\title{
Simulating Particle Dispersions in Nematic Liquid-Crystal Solvents
}

\author{
Ryoichi Yamamotot \\ Department of Chemistry, University of Cambridge, Lensfield Road, Cambridge CB2 1EW, UK
}

(October 29, 2018)

\begin{abstract}
A new method is presented for mesoscopic simulations of particle dispersions in nematic liquid crystal solvents. It allows efficient first-principle simulations of the dispersions involving many particles with many-body interactions mediated by the solvents. A simple demonstration is shown for the aggregation process of a two dimensional dispersion.

PACS numbers: 61.30.Cz, 61.30.Jf, 61.20.Ja
\end{abstract}

Dispersions of small particles in host fluids such as colloidal suspensions and emulsions are of considerable technological importance, and often appear in our everyday life in paints, foods, and drugs. Many kinds of exotic interactions between particles mediated by the host fluids are possible, including screened Coulombic [1], depletion [1], fluctuation induced [2], and surface induced [3] forces. A striking example occurs when spherical particles are immersed in a liquid-crystal solvent in the nematic phase. For a single particle, the orientation of the solvent molecules is distorted due to the anchoring of the solvent molecules at the particle surface. Extensive studies have been done on this effect, and several characteristic configurations of the nematic field around a spherical particle have been identified [4 9]. When the strength of anchoring is increased so that normal anchoring is preferred, the solvent changes from quadrupolar to dipolar symmetries around the particle. When more than two particles are immersed in the solvent, long-range anisotropic interactions are induced between particles due to elastic deformations of the nematic field [10 13. The anisotropic interactions can have a pronounced effect not only on the local correlations of the particles [10], but also on their phase behavior 14 17] and on their mechanical properties [15].

Since analytical approaches for investigating these kinds of complex materials are extremely difficult, computer simulations are essential to investigate their static and dynamical properties. In most dispersions, the host fluid molecules are much smaller and move much faster than the dispersed particles. This enables us to assume that the host fluid is in local equilibrium for any given particle configurations, and thus it is usually a good idea to use some coarse grained mesoscopic descriptions for the host fluids rather than treating them as fully microscopic molecules [18]. In the case of charged colloidal suspensions, a mesoscopic method for the first principle simulations can be derived by treating the counter ions as a charge density [19]. For the particle dispersions in nematic solvents considered here, the mesoscopic coarse grained free energy for the nematic solvent is well known to be the Frank free energy [20], and the total free en- ergy $\mathcal{F}$ of the system consists of the following two parts; the bulk term $\mathcal{F}_{e l}$ which presents elastic energy of the nematic and the surface term $\mathcal{F}_{s}$ which determines anchoring of the nematic field at the particle surface. Let $\mathbf{n}(\mathbf{r})$ be the director, a common direction on which solvent molecules are aligned on average with a constraint $|\mathbf{n}(\mathbf{r})|=1$. $\mathcal{F}$ can be given by functionals of $[\mathbf{n}(\mathbf{r})]$ for a given particle configuration $\left\{\mathbf{R}_{1} \cdots \mathbf{R}_{N}\right\}$;

$$
\begin{aligned}
& \mathcal{F}\left([\mathbf{n}(\mathbf{r})] ;\left\{\mathbf{R}_{1} \cdots \mathbf{R}_{N}\right\}\right) \\
& =\frac{K}{2} \int d \mathbf{r}\left[(\nabla \cdot \mathbf{n})^{2}+(\nabla \times \mathbf{n})^{2}\right]+\frac{W}{2} \oint d S\left[1-(\mathbf{n} \cdot \boldsymbol{\nu})^{2}\right],
\end{aligned}
$$

where $K$ is the Frank constant with the single elastic constant approximation, $W$ is the surface anchoring constant, and $\boldsymbol{\nu}$ is the unit vector normal to the colloidal surface [6,7]. The saddle-splay elastic term [7] is not considered in Eq.(11). The integral in the first term, $\mathcal{F}_{e l}$, runs over the whole solvent volume excluding the particles, and that in the second term, $\mathcal{F}_{s}$, runs over all solvent-particle interfaces. A simple scaling argument tells us $\mathcal{F}_{e l} \propto K a^{d-2}$ and $\mathcal{F}_{s} \propto W a^{d-1}$ with $a$ and $d$ being the particle radius and the system dimension, respectively, thus the physics should be determined by the ratio $\mathcal{F}_{s} / \mathcal{F}_{\text {el }} \propto W a / K$. Although this type of free energy functional is sufficient for performing Monte Carlo simulations where only values of $\mathcal{F}$ are needed for a given particle configurations, it is not useful for molecular dynamics (MD) or Brownian type simulations because the coupling between solvent and the particles is given implicitly by limiting the integration space in both $\mathcal{F}_{e l}$ and $\mathcal{F}_{s}$. This produces mathematical singularities at the interface when one calculates the force, $\mathbf{f}_{i}^{P S}=-\partial \mathcal{F} / \partial \mathbf{R}_{i}$, acting on each particle mediated by the nematic solvents. Calculating the force is crucial for performing efficient simulations of many particle systems. Another serious problem of this type of functional is that in order to give correct boundary conditions at the particle-solvent interface, one has to use appropriate coordinates for performing grid-based numerical simulations rather than the usual Cartesian coordinates. This is generally difficult for particles with non-spherical shapes or for systems involving many particles even when each particle has a spheri- 
cal shape. Also this makes the use of the periodic boundary condition difficult.

To overcome these problems, we have modified Eq.(1) by using a smooth interface between the solvent and the particles so that the coupling is given explicitly in the integrants through the interface. The new free energy functional we propose is

$$
\begin{aligned}
\mathcal{F} & \left([\mathbf{q}(\mathbf{r})] ;\left\{\mathbf{R}_{1} \cdots \mathbf{R}_{N}\right\}\right) \\
& =\frac{K}{4 R_{c}^{2}} \int d \mathbf{r}\left(1-\sum_{i=1}^{N} \phi_{i}(\mathbf{r})\right) \tanh \left[R_{c}^{2}\left(\nabla_{\alpha} q_{\beta \gamma}\right)^{2}\right] \\
& +\frac{W \xi}{2} \int d \mathbf{r} \sum_{i=1}^{N}\left[\frac{d-1}{d}\left(\nabla_{\alpha} \phi_{i}\right)^{2}-\left(\nabla_{\alpha} \phi_{i}\right)\left(\nabla_{\beta} \phi_{i}\right) q_{\alpha \beta}\right],
\end{aligned}
$$
(2)

where $\alpha, \beta, \gamma \in x, y, z$ and the summation convention is used. The explicit form of the interfacial profile $\phi_{i}$ between dispersed particles and solvents is given by

$$
\phi_{i}(\mathbf{r})=\frac{1}{2}\left(\tanh \frac{a-\left|\mathbf{r}-\mathbf{R}_{i}\right|}{\xi}+1\right),
$$

with the particle radius $a$ and the interface thickness $\xi$. Note that this reduces to Eq.(11) if $R_{c}, \xi \rightarrow 0$. Very recently, a similar idea of using smooth interface was proposed for treating the hydrodynamic forces acting on particles dispersed in simple liquids [21. In our case, the free energy is given by functionals of a traceless and symmetric second-rank tensor $q_{\alpha \beta}(\mathbf{r})=$ $n_{\alpha}(\mathbf{r}) n_{\beta}(\mathbf{r})-\delta_{\alpha \beta} / d$ rather than the director $\mathbf{n}(\mathbf{r})$ to take into account the symmetry of the nematic director $+\mathbf{n} \leftrightarrow-\mathbf{n}$ automatically. The semi-empirical functional form $1 / R_{c}^{2} \tanh \left[R_{c}^{2} \cdots\right]$ is applied in Eq.(2) to avoid the mathematical divergence of the elastic free energy density at the defect centers and to limit its value to $\Delta f \sim K / R_{c}^{2}$, which is the correct energy density difference between isotropic and nematic states, in the defect core regions of size $R_{c}$. Another way to avoid the divergence would be to use the Landau-de Gennes type free energy with an order parameter $Q_{\alpha \beta}(\mathbf{r})=Q(\mathbf{r}) q_{\alpha \beta}(\mathbf{r})$, but this requires a prohibitively small lattice spacing near the defect points 22.

The simulation procedure is as follows. i) For a given particle configuration $\left\{\mathbf{R}_{1} \cdots \mathbf{R}_{N}\right\}$, we obtain the interface profile $\phi_{i}(\mathbf{r})$ by Eq.(3). Then we can calculate the stable (or meta-stable) nematic configurations $\left[q_{\alpha \beta}^{(0)}(\mathbf{r})\right]$ which satisfy the equilibrium condition

$$
\left.\frac{\delta \mathcal{F}}{\delta q_{\alpha \beta}(\mathbf{r})}\right|_{\left[q_{\alpha \beta}(\mathbf{r})\right]=\left[q_{\alpha \beta}^{(0)}(\mathbf{r})\right]}=0
$$

under the director constraint $\left(n_{\alpha}(\mathbf{r})\right)^{2}=1$. One can perform this by numerical iterations such as the steepest descent or the conjugate gradient method. ii) Once $\left[q_{\alpha \beta}^{(0)}(\mathbf{r})\right]$ is obtained, the force acting on each particle mediated by the nematic solvents follows directly from the Hellmann-Feynman theorem,

$$
\begin{aligned}
\mathbf{f}_{i}^{P S} & \left(\left\{\mathbf{R}_{1} \cdots \mathbf{R}_{N}\right\}\right) \\
& =-\frac{\partial \mathcal{F}\left(\left[q_{\alpha \beta}^{(0)}(\mathbf{r})\right] ;\left\{\mathbf{R}_{1} \cdots \mathbf{R}_{N}\right\}\right)}{\partial \mathbf{R}_{i}} \\
& =\frac{K}{4 R_{c}^{2}} \int d \mathbf{r} \frac{\partial \phi_{i}}{\partial \mathbf{R}_{i}} \tanh \left[R_{c}^{2}\left(\nabla_{\alpha} q_{\beta \gamma}^{(0)}\right)^{2}\right] \\
& +W \xi \int d \mathbf{r} \frac{\partial\left(\nabla_{\alpha} \phi_{i}\right)}{\partial \mathbf{R}_{i}}\left(\nabla_{\beta} \phi_{i}\right) q_{\alpha \beta}^{(0)}
\end{aligned}
$$

This form is very convenient because we can compute both $\partial \phi_{i} / \partial \mathbf{R}_{i}$ and $\partial\left(\nabla_{\alpha} \phi_{i}\right) / \partial \mathbf{R}_{i}$ at any time since $\phi_{i}$ is an analytical function of $\mathbf{R}_{i}$. iii) Finally, we update the particle positions according to appropriate equations of motion such as

$$
m_{i} \frac{d^{2} \mathbf{R}_{i}}{d t^{2}}=\mathbf{f}_{i}^{P P}+\mathbf{f}_{i}^{P S}+\mathbf{f}_{i}^{H}+\mathbf{f}_{i}^{R},
$$

where $\mathbf{f}_{i}^{P P}$ is the force due to direct particle-particle interactions (hard or soft sphere for instance), $\mathbf{f}_{i}^{H}$ and $\mathbf{f}_{i}^{R}$ are the hydrodynamic and random forces. Repeating the steps i) iii) enables us to perform first-principles mesoscopic simulations for the dispersions containing many particles without neglecting many-body interactions.

We have performed simple simulations for a two dimensional (2D) system to demonstrate our simulation procedure. The system has $100 \times 100$ lattice sites in a square box with a linear length $L=100$. Other physical parameters are chosen rather arbitrarily as $R c=1$, $a=5$, and $\xi=2$, where the unit of length is the lattice spacing $l$. Since the nematic configurations in 2D can be expressed by a single scalar field $[\theta(\mathbf{r})]$, the tilt angle of the director against the horizontal $(x-)$ direction, Eq.(田) then reduces to

$$
\frac{\delta \mathcal{F}}{\delta \theta(\mathbf{r})}=\frac{\partial q_{\alpha \beta}(\mathbf{r})}{\partial \theta(\mathbf{r})} \frac{\delta \mathcal{F}}{\delta q_{\alpha \beta}(\mathbf{r})}=0
$$

with $q_{x x}=\cos ^{2} \theta-1 / 2, q_{y y}=\sin ^{2} \theta-1 / 2$, and $q_{x y}=$ $q_{y x}=\cos \theta \sin \theta$. The boundary condition is fixed at $\theta(\mathbf{r})=0$ at the edge of the box to avoid rotations of the reference frame. We first calculated stable nematic configurations around a single particle for different $W a / K$, and found two stable configurations. The first configuration, which we refer to as weak anchoring, contains no topological defect. In the second configuration, which we refer to as strong anchoring, the particle is accompanied by two $-1 / 2$ charge point defects. Typical examples of the weak and strong anchoring are shown in Fig.1(a) with $W a / K=2$ and Fig.1(b) with $W a / K=4$, respectively. The distance between the defects and the particle center is about $1.3 a$. We note that both configurations possess quadrupolar symmetries, and the later would correspond to the Saturn ring configuration in three dimensional (3D) systems. Although in principle particles can be accompanied by one -1 charge hedgehog defect in 
$2 \mathrm{D}$ as well as in $3 \mathrm{D}$, such configurations are unstable in the present $2 \mathrm{D}$ system since the elastic penalty of having $m$ point defects with charge $c$ scales as $m K c^{2}$. This was directly confirmed by recent simulations with perfect normal anchoring [22] and also by our simulations. The total free energies $\mathcal{F} / W$ are plotted in Fig.2 as functions of $W a / K$. While both configurations can coexist in the narrow transition regime $2.2 \leq W a / K \leq 2.5$, our model predicts a clear first-order transition from the weak anchoring to the strong anchoring around $W a / K \simeq 2.3$.

We next simulated the aggregation and ordering process of 30 colloidal particles after the isotropic to nematic transition of the solvent occurred. Here we used the periodic boundary condition and set $W a / K=4$ so that each particle is accompanied by two $-1 / 2$ charge defects. Other parameters are the same as in the previous single particle case. The simulation was performed starting from a random particle configuration which is a typical configuration when the solvent is in the isotropic phase $(K=0)$. We then set $K=1$ and calculated $\mathbf{f}_{i}^{P S}$ according to the present procedure. The particle configurations were updated by numerically solving the steepest descent equation,

$$
\zeta \frac{d \mathbf{R}_{i}}{d t}=\mathbf{f}_{i}^{P S}+\mathbf{f}_{i}^{P P},
$$

which is obtained by simply substituting $d^{2} \mathbf{R}_{i} / d t^{2}=0$, $\mathbf{f}_{i}^{R}=0$, and $\mathbf{f}_{i}^{H}=-\zeta d \mathbf{R}_{i} / d t$ in Eq.(7). $\quad \zeta=1$ is a friction constant and thus the off-diagonal components of the hydrodynamic interaction was not considered. Here we obtain $\mathbf{f}_{i}^{P P}=-\partial E_{P P} / \partial \mathbf{R}_{i}$ from the repulsive part of the Lennard-Jones potential, $E_{P P}=$ $0.4 \sum_{i=1}^{N-1} \sum_{j=i+1}^{N}\left[\left(2 a /\left|\mathbf{r}_{i}-\mathbf{r}_{j}\right|\right)^{12}-\left(2 a /\left|\mathbf{r}_{i}-\mathbf{r}_{j}\right|\right)^{6}+1 / 4\right] *$ truncated at the minimum distance $\left|\mathbf{r}_{i}-\mathbf{r}_{j}\right|=2^{7 / 6} a$, to avoid the particles overlapping each other within the core radius $\simeq a$. Snapshots from the present simulation are shown in Fig.3(a) for an aggregation stage, and in Fig.3(b) at a later time, where the particles are forming ordered clusters due to the anisotropic attractions between them. Note is added that only up to two particle simulations have been done so far [7] and simulations of more than three particles would be extremely difficult or almost impossible by other methods ever proposed.

In summary, we have developed an extremely powerful simulation method to investigate particle dispersions interacting via anisotropic solvents. We proposed a free energy functional which is suitable for MD type simulations. The following modifications have been made to the usual Frank free energy functional. i) The free energy is given by a functional of a tensor $\mathbf{q}$ rather than a vector $\mathbf{n}$ to take into account symmetry of the nematic director $+\mathbf{n} \leftrightarrow-\mathbf{n}$. ii) The coupling between the nematic solvent and particles at the interfaces is introduced explicitly through a smooth interface so that we can analytically calculate the force acting on each particle mediated by the host by taking derivatives of the free energy according to the particle positions. iii) The value of the free energy density is limited semi-empirically to avoid a mathematical divergence in the defect centers. We have performed demonstrations for a 2D dispersion and confirmed that the method works well even when the system contains point defects. Applications of this method to 3D systems should have no theoretical difficulties, but require heavier computation. This should allow the simulation of the chaining of the particles caused by the possible dipolar symmetry of the nematic configurations around a single particle. Although we have shown only simple demonstrations of the method by performing simulations of the $2 \mathrm{D}$ system in this letter, simulations with physically more interesting situations such as systems with non-circular particles, asymmetric particle pairs with different particle size, or particles with non-normal anchoring as well as more realistic simulations in 3D systems are now underway.

The author thanks Prof. J.P. Hansen and Dr. A. Louis for helpful discussions. He acknowledges also the Ministry of Education, Culture, Sports, Science and Technology of Japan for supporting his stay in Cambridge in 2000/2001. Calculations have been carried out at the Human Genome Center, Institute of Medical Science, University of Tokyo, and the Supercomputer Center, Institute of Solid State Physics, University of Tokyo.

Permanent address: Department of Physics, Kyoto University, Kyoto 606-8502 Japan.

Email: ryoichi@scphys.kyoto-u.ac.jp

[1] W.B. Russel, D.A. Saville, and W.R. Schowalter, Colloidal Dispersions (Cambridge University Press, Cambridge, 1995).

[2] V.M. Mostepanenko and N.N. Trunov, The Casimir Effect and its Application (Clarendon Press, Oxford, 1997).

[3] A. Borštnik, H, Stark, and S. Žumer, Phys. Rev. E 60, 4210 (1999).

[4] E.M. Terentjev, Phys. Rev. E 51, 1330 (1995).

[5] S. Ramaswamy, R. Nityananda, V.A. Raghunathan, and J. Prost, Mol. Crys. Liq. Crys. 288, 175 (1996).

[6] R.W. Ruhwandl and E.M. Terentjev, Phys. Rev. E 56, 5561 (1997).

[7] H. Stark, Eur. Phys. J. B 10, 311 (1999); H. Stark, J. Stelzer, and R. Bernhard, Eur. Phys. J. B 10, 515 (1999).

[8] O. Mondain-Monval, J.C. Dedieu, T. Gulik-Krzywicki, and P. Poulin, Eur. Phys. J. B 12, 567 (1999).

[9] Y.G. Gu and N.L. Abbott, Phys. Rev. Lett. 85, 4719 (2000).

[10] P. Poulin, H. Stark, T.C. Lubensky, and D.A. Weitz, Science 275, 1770 (1997); P. Poulin, V. Cabuil, and D.A. Weitz, Phys. Rev. Lett. 79, 4862 (1997); P. Poulin and D.A. Weitz, Phys. Rev. E 57, 626 (1998). 
[11] R.W. Ruhwandl and E.M. Terentjev, Phys. Rev. E 55, 2958 (1997).

[12] T.C. Lubensky, D. Pettey, N. Currier, and H. Stark, Phys. Rev. E 57, 610 (1998).

[13] B.I. Lev and P.M.Tomchuk, Phys. Rev. E 59, 591 (1999).

[14] V.A. Raghunathan, P. Richetti, and D. Roux, Langmuir 12, 3789 (1996); V.A. Raghunathan, P. Richetti, D. Roux, F. Nallet, and K. Sood, Langmuir 16, 4720 (2000).

[15] S.P. Meeker, W.C.K. Poon, J. Crain, and E.M. Terentjev, Phys. Rev. E 61, R6083 (2000); V.J. Anderson, E.M. Terentjev, S.P. Meeker, J. Crain, and W.C.K. Poon, Eur. Phys. J. E 1, 11 (2001); V.J. Anderson, E.M. Terentjev, Eur. Phys. J. E 1, 21 (2001).

[16] J.C. Loudet, P. Barios, and P. Poulin, Nature 407, 611 (2000).

[17] J. Yamamoto and H. Tanaka, Nature 409, 321 (2001).

[18] J.L. Billeter and R.A. Pelcovits, Phys. Rev. E 62, 711 (2000).

[19] H. Löwen, P.A. Madden, and J.P Hansen, Phys. Rev. Lett. 68, 1081 (1992); H. Löwen, J.P Hansen, and P.A. Madden, J. Chem. Phys. 98, 3275 (1993).

[20] P.G. de Gennes and J. Prost, The Physics of Liquid Crystals, 2nd ed. (Clarendon, Oxford, 1993).

[21] H. Tanaka and T. Araki, Phys. Rev. Lett. 85, 1338 (2000).

[22] J. Fukuda and H. Yokoyama, Eur. Phys. J. E 4, 389 (2001). 

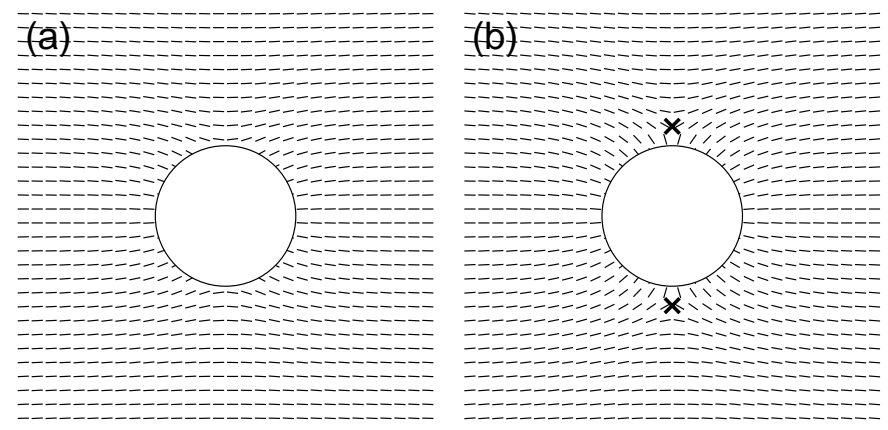

FIG. 1. Director configurations around a single particle for (a) weak anchoring case without defect obtained at $W a / K=2$ and (b) strong anchoring case accompanied with two $-1 / 2$ charge point defects indicated with the crosses obtained at $W a / K=4$. The white disks indicate the particles with radius $a=5$. Only $9 \%$ of the total system is shown for display purpose.

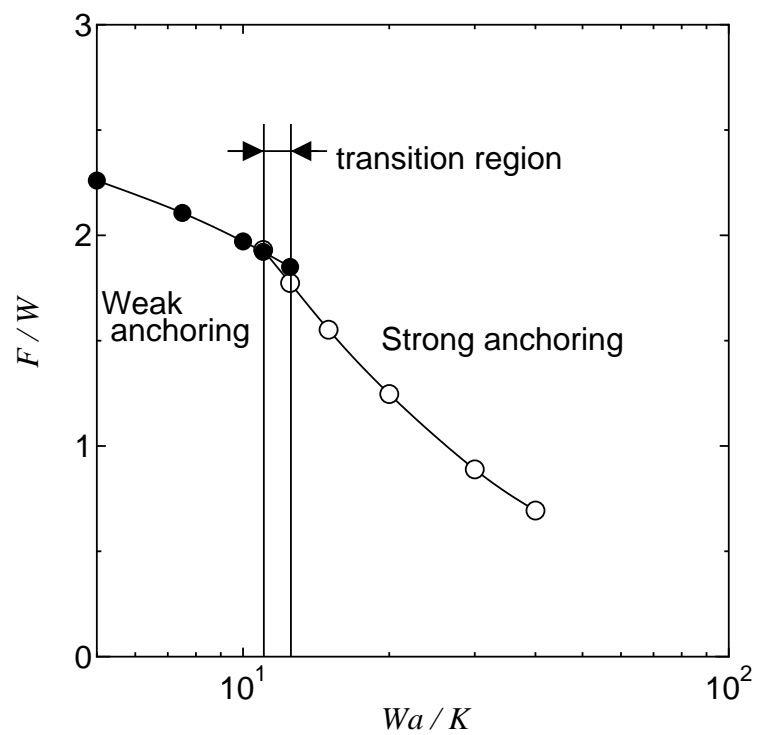

FIG. 2. The total free energies for the single particle cases as functions of the strength of the anchoring constant $W a / K$. Our model predicts a first-order transition from weak to strong anchoring around $W a / K \simeq 2.3$.
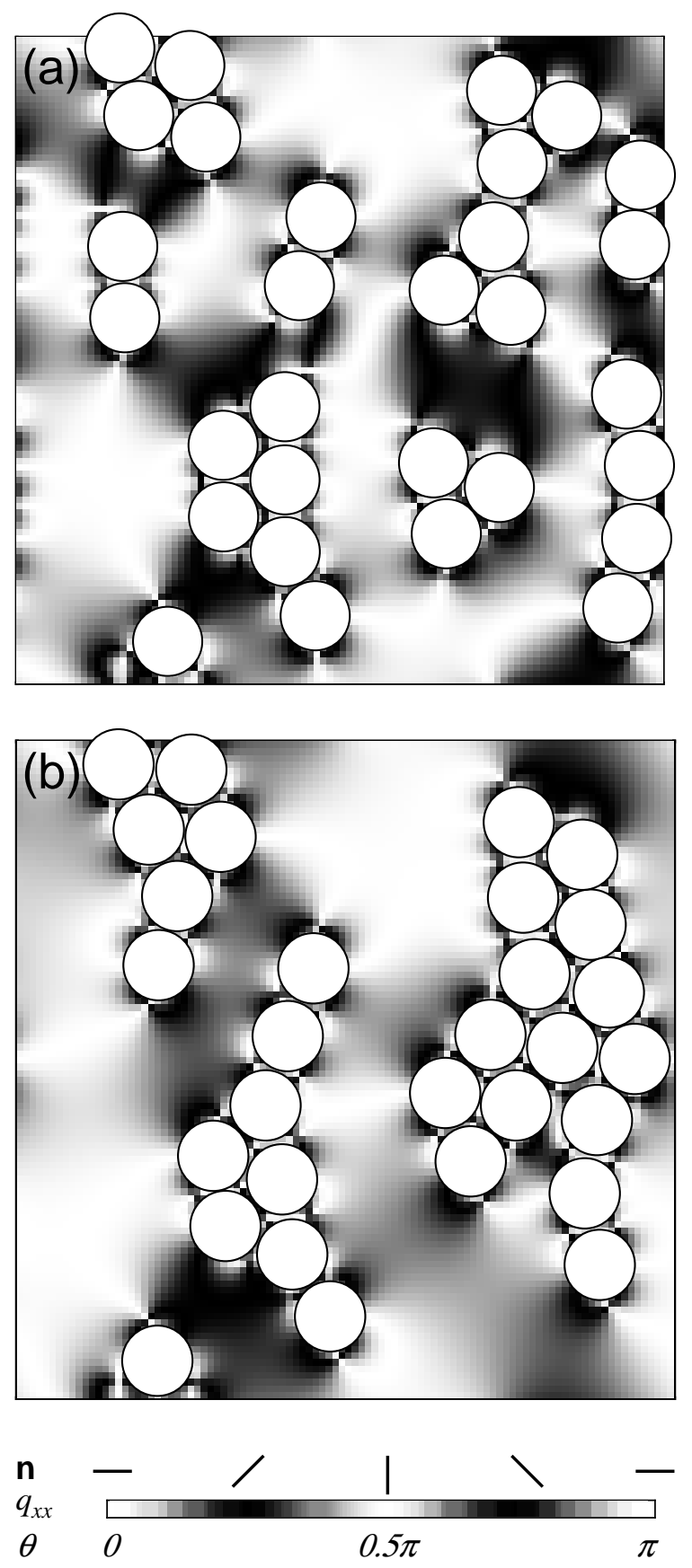

FIG. 3. The aggregation and ordering process of colloidal particles when the solvent exhibit the isotropic $(K=0)$ to nematic $(K=1)$ phase transition at $t=0$. Snapshots (a) in an aggregation stage $(t=10)$ and $(\mathrm{b})$ at a later time $(t=100)$, where ordering of the particles are observed. Each particle is accompanied by two $-1 / 2$ charge point defects. Darkness presents the value of $q_{x x}^{2}$. Black and white correspond to $q_{x x}^{2}=0$ and 0.25 , respectively. Those correspond also to $\theta=0.25 \pi, 0.75 \pi$ and $\theta=0,0.5 \pi, \pi$ as shown in the gradation map. 